In the manuscript "Risk and preventive factors for traffic accidents: Analysis of children's perception using the edutherapeutic method”, DOI: 10.1590/1984-0462/2020/38/2018281, published in the Rev Paul Pediatr. 2020;38:e2018281, on page 7:

\title{
Where it reads:
}

Funding

This study did not receive funding.

\section{It should read:}

Funding

This study was financed in part by the Coordenação de Aperfeiçoamento de Pessoal de Nível Superior - Brasil (CAPES) - Finance Code 001. 